\title{
Parallel BP Neural Network on Single-chip Cloud Computer
}

\author{
Boyang Li Chen Liu \\ Department of Electrical and Computer Engineering \\ Clarkson University \\ Potsdam, New York, USA \\ \{boyli, cliu\}@clarkson.edu
}

\begin{abstract}
Neural network has been a clear focus in machine learning area. Back propagation (BP) method is frequently used in neural network training. In this work we paralleled BP neural network on Single-Chip Cloud Computer (SCC), an experimental processor created by Intel Labs, and analyzed multiple metrics under different configurations. We also varied the number of neurons (nodes) in the hidden layer of the BP neural network and studied the impact. The experiment results show that a better performance can be obtained with SCC, especially when there are more nodes in the hidden layer of BP neural network. A low voltage and frequency configuration contributes to a low power per speedup. What is more, a medium voltage and frequency configuration contributes to both a low energy consumption and energy-delay product.
\end{abstract}

Keywords- Back Propagation; Neural Netork; Single-chip Cloud Computer; Energy-Aware Computing; Power-Aware Computing

\section{INTRODUCTION}

Artificial Neural Network (ANN) has been widely adopted in areas such as handwriting recognition, voice recognition, and image classification. Back propagation (BP) neural network is a common way to train ANN combined with gradient descent to minimize the difference between the actual output and the desired output of ANN by adjusting the weights of the connection in the network.

On the other hand, the current revolution in hardware towards multi-core and many-core platforms presents a huge challenge to software applications. Correspondingly, the Single-Chip Cloud Computer (SCC) experimental processor is a 48-core "concept vehicle" created by Intel Labs as a platform for many-core software research [1]. To the best of our knowledge, this is the first effort of its kind to port the BP neural network onto the SCC platform. Because the execution time, energy consumption and energy-delay product of parallel BP neural network will differ with different number of nodes in the hidden layer, BP neural network with 5 nodes, 10 nodes, 20 nodes in the hidden layer are experimented, respectively. Our objective is to observe the relationship among execution time, power and energy consumption, power per speedup (PPS), energydelay product (EDP), and the number of cores under different configurations.

The rest of the paper is structured as follows. Section II gives a brief introduction to SCC platform as well as the BP neural network and related work. Section III presents the experiment configurations and how to parallel the BP neural network program on SCC. Section IV analyzes the experiment results. Finally, the conclusions and future work are summarized in Section V.

\section{BACKGROUND}

\section{A. Single-Chip Cloud Computer}

The Single-Chip Cloud Computer is a many-core research chip, which is provided by Intel Labs to study many-core CPUs, their architectures as well as the techniques used to program them. The Single-Chip Cloud Computer contains 48 Pentium $^{\mathrm{TM}}$ class IA- 32 cores on a $6 \times 4$ 2D-mesh network of tiled core clusters with high-speed I/Os on the periphery [2], as shown in Figure 1. Every tile consists of two cores and a router shared by the two cores. The 48 cores are divided into 6 voltage domains and 24 frequency domains on the SCC chip (the routers and other peripherals are on separate voltage and frequency domains). As shown in Figure 2, every four tiles share a power domain, and the programmer can change the voltage for all the cores in a voltage domain. Every two cores on the same tile can also have their own frequency within the frequency domain [3].

The SCC provides the capability of changing control voltage and frequency of the cores separately. This ability supports research on power-aware computing applications. SCC actually is the predecessor of the more recent Intel Many Integrated Core (MIC) architecture [4]. The reason in this work we choose SCC as the experiment platform is because of its versatile capability in terms of dynamic voltage and frequency scaling (DVFS), which allows us manipulate the voltage and frequency directly at the application level. This suits the purpose of this study very well.

\section{B. BP Neural Network}

BP neural network is widely used in machine learning and artificial neural network areas. The errors propagate backwards from the output nodes to the input nodes, as shown in Figure 3. Then the gradient of the error of the network about the network's modifiable weights is calculated [5]. Finally, the stochastic gradient descent algorithm uses this gradient to find weights that will finally minimize the error.

If the weight is updated every time after one input sample, the performance of the network would depend on the input sequence of the samples to a large degree. To eliminate the impact of the input sequence of the samples, batch learning is a good choice. In batch training, the training and working phases are separate [6]. Hence, batch 


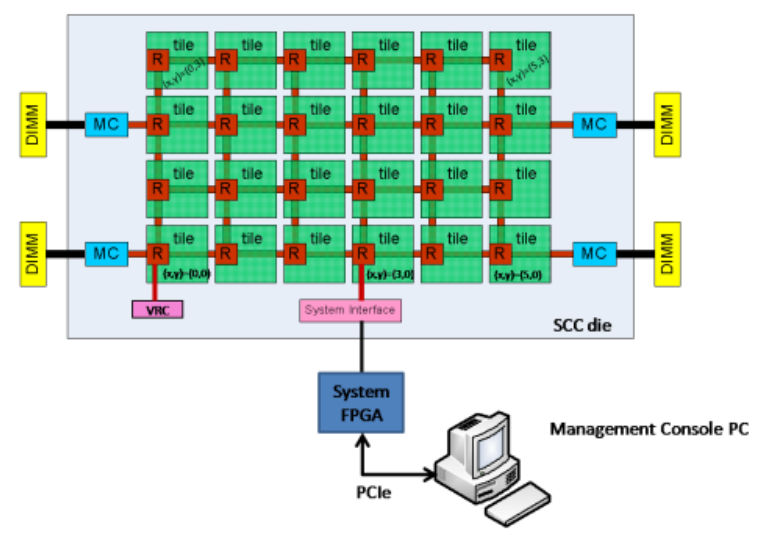

Figure 1. SCC Top-Level Architecture [3]

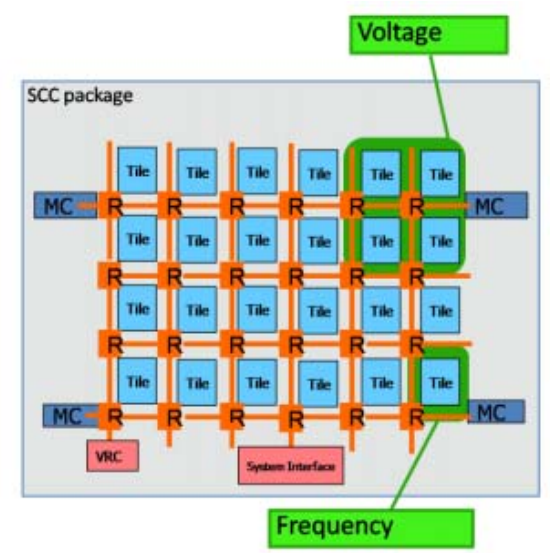

Figure 2. Voltage and frequency domain on the SCC [3]

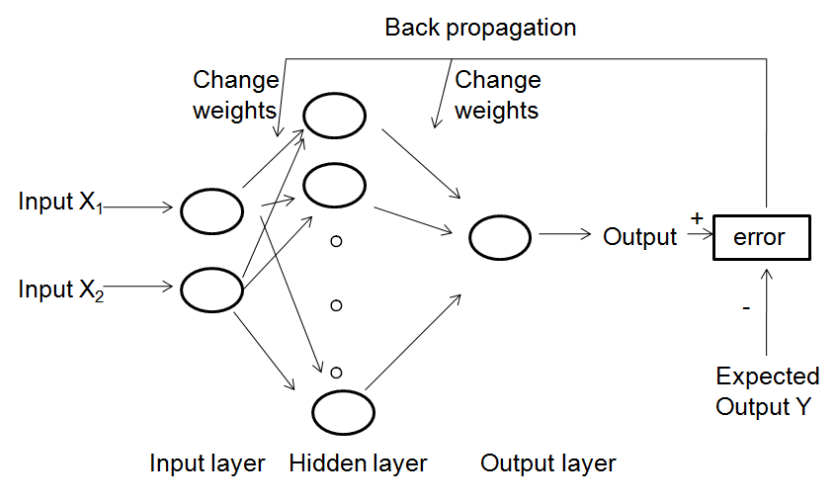

Figure 3. A typical back propagation neural network architecture of two inputs, one output and one hidden layer

learning is employed in this work.

\section{Related Work}

Due to the versatile capability of SCC in terms of DVFS, many applications have already been ported onto SCC platform. Torres et al. implemented iris matching on SCC platform [7]. In their study, many energy-related metrics such as power, energy, EDP, and PPS are measured. Also, there are researches on BP neural network on other multi- core platforms. Lopes et al. implemented the multiple backpropagation algorithms on Graphic Processing Unit (GPU) platform. Their work shows a reduction in computation cost compared with the standalone CPU version [8]. In addition, Rodopoulos et al. used SCC to simulate a biologically accurate neuron [9]. In this work, we want to implement the BP neural network on SCC platform. In the meantime, we want to use the DVFS capability of SCC to measure energyrelated metrics.

\section{Methodology}

\section{A. Experiment Configuration}

BP neural network built in this work has two input variables, one hidden layer, one output layer and one output variable. In the experiment, we try to train this network to learn the operation of addition. The number of training samples is set to 9600 . Since there are two input variables, too many neurons (nodes) in the hidden layer will lead to an over-fitting neural network. The numbers of nodes that we use in hidden layer are 5, 10, and 20, respectively. The configurations in terms of frequency/voltage for every power domain on SCC are set to $0.8 \mathrm{~V} / 400 \mathrm{MHz}$ (low), $0.9 \mathrm{~V} / 533 \mathrm{MHz}$ (medium), and $1.2 \mathrm{~V} / 800 \mathrm{MHz}$ (high), respectively. The number of cores that we use in the experiment is $1,2,4,8,16,32$, and 48 , separately.

\section{B. Experiment Procedure}

There are two widely used parallel approaches: task parallelism and data parallelism. In task parallelism, we partition the various tasks carried out in solving the problem among the cores. In data parallelism, we partition the data used in solving the problem among the cores and each core carries out more or less similar operations on its part of the data [10]. In our experiment, data parallelism is employed. The procedure of the experiment is described as follows:

1) The samples are sent to different cores according the ID of cores, starting from Core 0 all the way to Core 47. This means Core $n$ deals with sample $n, n+8, n+16, \ldots$, so on and so forth.

2) In every entire presentation of the training data (an epoch), every core calculates its total change of each weight and the average loss of the loss function. Then the total changes of weight of each core are added together. Similarly, the average losses of the loss function in each core are added together in order to calculate the average loss of loss function of all the cores.

3) Both the results are put in Core 0, then broadcast the results to all the participating cores.

4) We set the training times upper limit to 20000. We assume that the performance of neural network is satisfactory when the average error is less than 0.01. Thus, either on the condition that the times of training are not less than 20000 or that the average error is less than 0.01, training is stopped. Otherwise, the network would be trained with the new weights. We feel the essence of the limit that we put on training times and average error are the 
same, since both are to stop the training at the proper time to get a desired performance. If a neural network is trained for too many times, one side effect is that it may lead to over-fitting. If a neural network is trained not enough, the prediction may not be precise. Thus, we set the training times upper limit to 20000 and the bottom limit of average error to 0.01 .

\section{EXPERIMENTAL RESULTS}

The energy-related metrics we have measured are execution time, power consumption, power per speedup, energy consumption and energy-delay product. The execution time includes the communication time among cores, since the SCC follows the message-passing programming model. However, the overhead time that it takes to measure the power consumption has been deducted. Power consumption is obtained by measuring the voltage and current of the SCC chip only. Thus, the power reading does not include DDR DIMMs, which are off-chip. Power per speedup is calculated by dividing power consumption by the speedup achieved with increasing the number of cores. What is more, energy and EDP are calculated based on the power and execution time readings we get.

\section{A. Execution Time}

Figure 4 in log-log scale reflects the relationship between the execution time (in seconds) and the number of cores, while varying the number of nodes in the hidden layer of the BP neural network. The result shows that for a fixed number of nodes in the hidden layer, first, with cores increasing (from 1 core to 32 cores), the execution time decreases correspondingly; second, when the number of cores is fixed, the higher the frequency is, the less execution time the program takes. Overall, the high voltage and frequency setting gives the best performance.

Another interesting thing to observe is that with the number of nodes in hidden layer decreasing, the decreasing rate of execution time becomes slow when the number of cores increases from 32 to 48 . When the number of nodes in hidden layer is five, the execution time even increases with the number of cores increasing from 32 to 48 . The reason for that is when the number of nodes in the hidden layer is small, the percentage that the computation part takes in the whole program is small correspondingly. When the number of node is 5 , with the number of cores increasing from 32 to 48 , the time saved by distributing the workload in a parallel fashion even cannot make up the communication overhead caused among the cores.

\section{B. Power Consumption}

The experiment results show that no matter the number of nodes in hidden layer is 5,10 or 20, the power consumption (in watts) is identical, which means the number of nodes in the hidden layer has no effect on the power assumption. Thus, only the data of 5 nodes in hidden layer are presented here. Figure 5 in $\log -\log$ scale reflects the relationship between the power consumption (in watts)

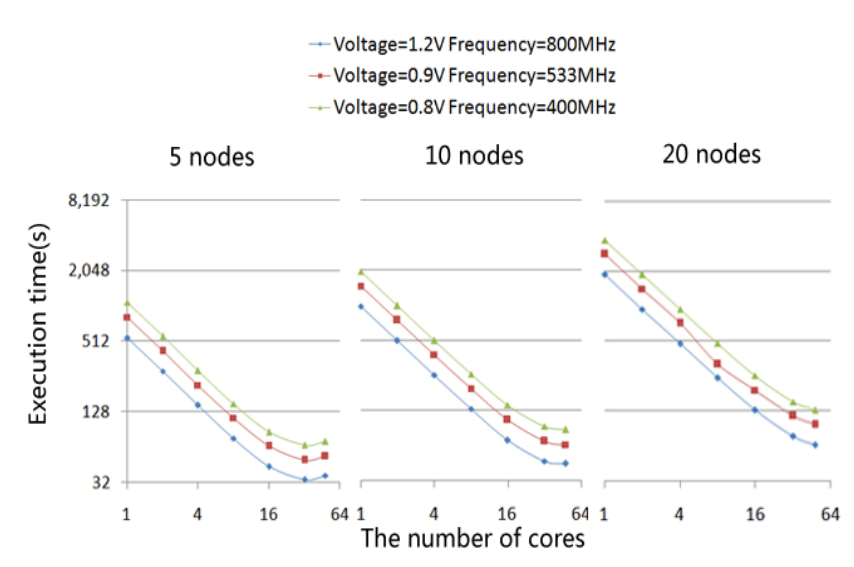

Figure 4. The relationship between execution time and the number of cores

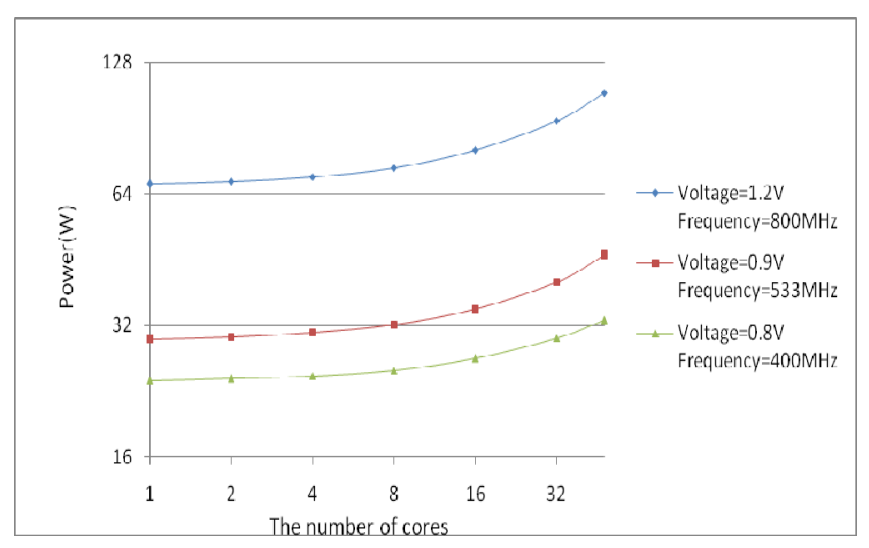

Figure 5. The relationship between power and the number of cores when the number of nodes in hidden layer is 5

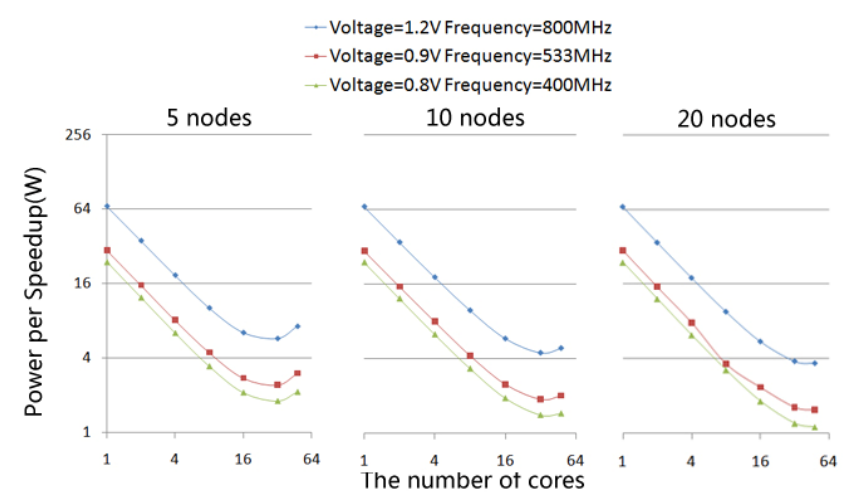

Figure 6. The relationship between power per speedup and the number of cores

and the number of cores. With the number of cores increasing, the increasing rate of power consumption becomes faster, as more power domains are involved in the computation. What is more, both the power consumption and the increasing rate of power consumption become larger as the voltage level increases. Overall, the low voltage and frequency setting gives the best power reading. 


\section{Power per Speedup}

Power per speedup (PPS) was proposed by Mair et al. [11] as an indicator to measure energy efficiency. This metric gives the power required for a given level of speedup through parallelism. Therefore, a small PPS value is desired. Figure 6 in log-log scale reflects the relationship between the power per speed up (in watts) and the number of cores. When the number of nodes in the hidden layer is fixed, configuration $(0.8 \mathrm{~V} / 400 \mathrm{MHz})$ provides the best PPS results for all core counts. So it is most power efficient to use the low voltage and frequency setting to achieve a given speedup when we increase the number of cores.

\section{Energy Consumption}

Figure 7 in log-log scale reflects the relationship between the energy consumption (in joules) and the number of cores, while varying the number of nodes in hidden layer. It can be seen that with the number of nodes in hidden layer increasing, the minimum of energy consumption will move in the direction where the number of cores increases. As is discussed above, the number of nodes in the hidden layer of the neural network has no effect on the power assumption. With the number of nodes in hidden layer increasing, the decreasing rate of execution time is greater than the increasing rate of the power consumption. Thus, for BP neural network, energy is more affected by execution time. The energy consumption curve's similarity to the execution time under each configuration also supports this view.

Even when the number of nodes in hidden layer is different, the energy consumption is always the highest under the configuration $(1.2 \mathrm{~V} / 800 \mathrm{MHz})$ when the number of cores is fixed. When the number of cores is small $(1,2,4$, or 8 cores), usually the energy consumption under the configuration $(0.9 \mathrm{~V} / 553 \mathrm{MHz})$ is smaller than the configuration $(0.8 \mathrm{~V} / 400 \mathrm{MHz})$. When the number of cores is medium (16 and 32 cores), the energy consumption under these two configurations is almost the same. When the number of cores is large (48 cores), the energy consumption under configuration $(0.8 \mathrm{~V} / 400 \mathrm{MHz})$ is smaller. The reason for that is when both the number of the cores and the number of the nodes in hidden layer are fixed, the slopes of the execution time in log-log scale under the configuration $(0.8 \mathrm{~V} / 400 \mathrm{MHz})(0.9 \mathrm{~V} / 533 \mathrm{MHz})$ or $(1.2 \mathrm{~V} / 800 \mathrm{MHz})$ are almost the same while the slopes of power in log-log scale decreases from the configuration $(1.2 \mathrm{~V} / 800 \mathrm{MHz})$ to the configuration $(0.8 \mathrm{~V} / 500 \mathrm{MHz})$. This change of the slopes is more significant as the number of cores increases.

\section{E. Energy-Delay Product}

Figure 8 in log-log scale reflects the relationship between the energy-delay product (in joules'seconds) and the number of cores, while varying the number of nodes in hidden layer. The experiment results show that the shape of the EDP curve for different number of nodes in the hidden layer is very similar to the energy curve shape. The minimum point of EDP will move in the same direction as the minimum point of energy moves. The difference is that when the number of nodes and the number of cores are

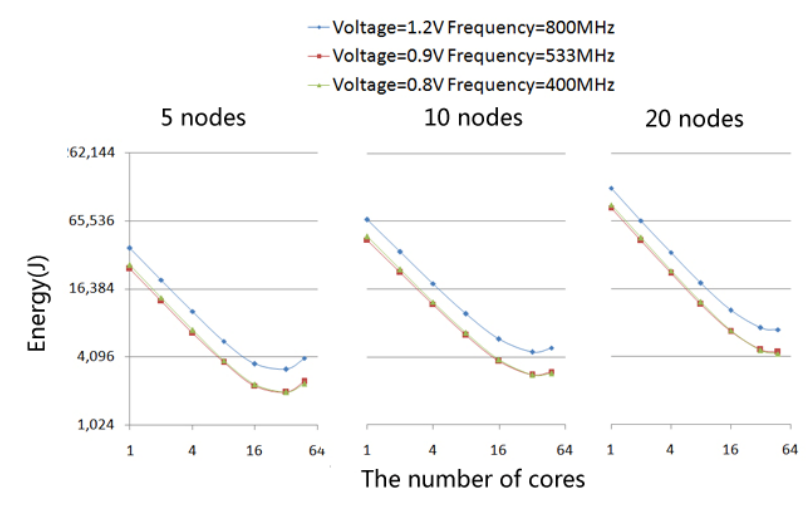

Figure 7. The relationship between energy and the number of cores

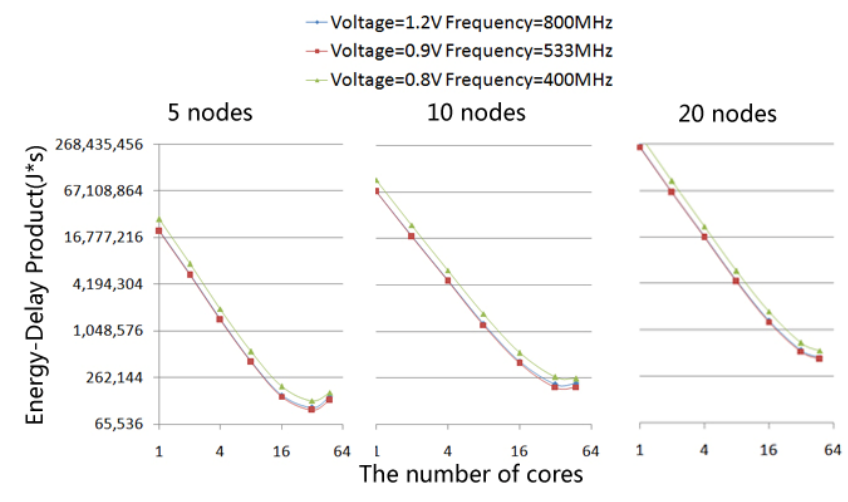

Figure 8 . The relationship between energy-delay product and the number of cores

TABLE I BEST CONFIGURATION FOR EACH METRIC

\begin{tabular}{|c|c|c|c|c|c|}
\hline \multirow{2}{*}{$\begin{array}{c}\text { Number } \\
\text { of nodes }\end{array}$} & \multicolumn{5}{|c|}{ Energy-related Metrics } \\
\cline { 2 - 6 } & $\begin{array}{c}\text { Execution } \\
\text { time }\end{array}$ & Power & PPS & Energy & EDP \\
\hline \multirow{2}{*}{5} & 32 cores & 1 cores & 32 cores & 32 cores & 32 cores \\
nodes & $1.2 \mathrm{~V} /$ & $0.8 \mathrm{~V} /$ & $0.8 \mathrm{~V} /$ & $0.8 \mathrm{~V} /$ & $0.9 \mathrm{~V} /$ \\
& $800 \mathrm{MHz}$ & $400 \mathrm{MHz}$ & $400 \mathrm{MHz}$ & $400 \mathrm{MHz}$ & $533 \mathrm{MHz}$ \\
\hline \multirow{2}{*}{10} & 48 cores & 1 cores & 32 cores & 32 cores & 32 cores \\
nodes & $1.2 \mathrm{~V} /$ & $0.8 \mathrm{~V} /$ & $0.8 \mathrm{~V} /$ & $0.8 \mathrm{~V} /$ & $0.9 \mathrm{~V} /$ \\
& $800 \mathrm{MHz}$ & $400 \mathrm{MHz}$ & $400 \mathrm{MHz}$ & $400 \mathrm{MHz}$ & $533 \mathrm{MHz}$ \\
\hline \multirow{2}{*}{20} & 48 cores & 1 cores & 48 cores & 48 cores & 48 cores \\
nodes & $1.2 \mathrm{~V} /$ & $0.8 \mathrm{~V} /$ & $0.8 \mathrm{~V} /$ & $0.8 \mathrm{~V} /$ & $0.9 \mathrm{~V} /$ \\
& $800 \mathrm{MHz}$ & $400 \mathrm{MHz}$ & $400 \mathrm{MHz}$ & $400 \mathrm{MHz}$ & $533 \mathrm{MHz}$ \\
\hline
\end{tabular}

constant, EDP under configuration $(0.9 \mathrm{~V} / 533 \mathrm{~Hz})$ is smallest, and EDP under configuration $(0.8 \mathrm{~V} / 400 \mathrm{MHz})$ is largest, though the EDP difference between configurations $(0.9 \mathrm{~V} / 533 \mathrm{~Hz})$ and $(1.2 \mathrm{~V} / 800 \mathrm{MHz})$ is very small. Overall, the medium voltage and frequency configuration gives us the best of both worlds when we taking both energy and user experience (in terms of system response time to finish the job) into consideration.

\section{F. Best Configuration for each Metric}

Table I summarizes the best configuration (number of cores and voltage/frequency) for each metric. 


\section{CONCLUSIONS}

With artificial neural network technique widely adopted across software applications, how to efficiently running this algorithm on emerging many-core platform has been of the interest for software developers. In this work, we studied the performance of back-propagation neural network on innovative Intel Single-Chip Cloud Computer platform using various metrics. We can conclude that paralleling BP neural network on SCC contributes to a better performance, especially with more nodes in the hidden layer. What is more, with the number of nodes increasing, minimum energy consumption and energy-delay product will move in the direction where the number of cores increases. A low voltage and frequency configuration contributes to a low power per speedup and a medium voltage and frequency configuration contributes to both a low energy consumption and energy-delay product. As far as future work is concerned, we are planning to port the BP neural network algorithm onto FPGA platform in a hardware accelerator approach, onto Intel Many Integrated Core (MIC) architecture, as well as Graphic Processing Unit (GPU) platform. Our vision is to perform a cross-platform comparison to identify the best architecture for this application. Other neural network design, such as deep neural network will also be considered.

\section{ACKNOWLEDGMENT}

We would like to thank Intel Labs for providing the Single-Chip Cloud Computer to conduct this research. We would like to thank Gildo Torres for his technical knowledge of the SCC platform. This work is supported by the National Science Foundation under Grant Numbers IIP1332046 and ECCS-1301953. Any opinions, findings, and conclusions or recommendations expressed in this material are those of the authors and do not necessarily reflect the views of the National Science Foundation. We would also like to thank the anonymous reviewers for their constructive feedbacks.
[1] T. G. Mattson, M. Riepen, T. Lehnig, P. Brett, W. Haas, P. Kennedy, J. Howard, S. Vangal, N. Borkar, G. Ruhl, and S. Dighe, "The 48core scc processor: the programmer's view," in Proceedings of the $2010 \mathrm{ACM} / \mathrm{IEEE}$ International Conference for High Performance Computing, Networking, Storage and Analysis, ser. SC '10. Washington, DC, USA: IEEE Computer Society, 2010, pp. 1-11. [Online]. Available:http://dx.doi.org/10.1109/SC.2010.53

[2] J.Howard, S.Dighe, Y.Hoskote, et al.,"A 48-Core IA-32 messagepassing processor with DVFS in 45nm CMOS," Solid- State Circuits Conference Digest of Technical Papers (ISSCC), 2010 IEEE International , pp.108-109, 7-11 Feb. 2010

[3] "SCC platform overview, " Intel Labs,

https://communities.intel.com/servlet/JiveServlet/previewBody/5512102-2-22524/SCC_Platform_Overview.pdf

[4] Intel Many Integrated Core Architecture - Advanced, http://www.intel.com/content/www/us/en/architecture-andtechnology/many-integrated-core/intel-many-integrated-corearchitecture.html

[5] Paul J. Werbos, "The roots of backpropagation. from ordered derivatives to neural networks and political forecasting," New York, NY: John Wiley \& Sons, Inc. ISBN: 0-471-59897-6

[6] Nick Littlestone, "From on-line to batch learning," COLT '89 Proceedings of the second annual workshop on Computational learning theory, pp 269-284, ISBN: 1-55860-086-8

[7] Gildo Torres, Jed Kao-Tung Chang, Fang Hua, Chen Liu and Stephanie Schuckers, "A Power-Aware Study of Iris Matching Algorithms on Intel's SCC," The 2013 International Workshop on Embedded Multicore Systems (ICPP-EMS 2013), in conjunction with ICPP 2013, Lyon, France, October 1-4, 2013, DOI:10.1109/ICPP.2013.122

[8] Noel Lopes, Bernardete Ribeiro, "GPU Implementation of the multiple back-propagation algorithm," Intelligent Data Engineering and Automated Learning - IDEAL 2009,Lecture Notes in Computer Science Volume 5788, 2009, pp 449-456

[9] D. Rodopoulos, G. Chatzikonstantis, A. Pantelopoulos, D. Soudris, C.I. De Zeeuw, C. Strydis, "Optimal mapping of inferior olive neuron simulations on the Single-Chip Cloud Computer", Embedded Computer Systems: Architectures, Modeling, and Simulation (SAMOS XIV), 2014 International Conference on, pp367-374, DOI: 10.1109/SAMOS.2014.6893235

[10] Peter Pacheco, "An Introduction to parallel programming", Morgan Kaufmann; 1st Edition (January 21, 2011) ISBN: 0123742609

[11] J.Mari, K. Leung, and Z. Huang, "Metrics and task scheduling policies for energy saving in multicore computers," in Grid Computing, $11^{\text {th }}$ IEEE/ACM International Conference on, IEEE, 2010, pp. 266-273.

\section{REFERENCES}

